\title{
Distributed Consensus-Based Weight Design for Cooperative Spectrum Sensing
}

\author{
Wenlin Zhang, Yi Guo, Senior Member, IEEE, Hongbo Liu, Yingying (Jennifer) Chen, \\ Zheng Wang, and Joseph Mitola III
}

\begin{abstract}
In this paper, we study the distributed spectrum sensing in cognitive radio networks. Existing distributed consensus-based fusion algorithms only ensure equal gain combining of local measurements, whose performance may be incomparable to various centralized soft combining schemes. Motivated by this fact, we consider practical channel conditions and link failures, and develop new weighted soft measurement combining without a centralized fusion center. Following the measurement by its energy detector, each secondary user exchanges its own measurement statistics with its local one-hop neighbors, and chooses the information exchanging rate according to the measurement channel condition, e.g., the signal-to-noise ratio (SNR). We rigorously prove the convergence of the new consensus algorithm, and show all secondary users hold the same global decision statistics from the weighted soft measurement combining throughout the network. We also provide distributed optimal weight design under uncorrelated measurement channels. The convergence rate of the consensus iteration is given under the assumption that each communication link has an independent probability to fail, and the upper bound of the iteration number of the $\epsilon$-convergence is explicitly given as a function of system parameters. Simulation results show significant improvement of the sensing performance compared to existing consensus-based approaches, and the performance of the distributed weighted design is comparable to the centralized weighted combining scheme.
\end{abstract}

Index Terms-Cooperative spectrum sensing, weighted average consensus, cognitive radio networks

\section{INTRODUCTION}

OGNITIVE radio (CR) [1] aims to improve the spectrum utilization by allowing unlicensed secondary user (SU) to operate in the 'white spaces' of the licensed spectrum bands without interfering the licensed primary user (PU). Revealing a future communication paradigm with dramatically enhanced spectrum efficiency, cognitive radio network is also referred as the neXt Generation (XG) or dynamic spectrum access (DSA) network [2].

One of the fundamental techniques in cognitive radio is spectrum sensing, which enables the secondary users to detect the presence of a primary user in the spectrum, see [3], [4] and the references therein. The main challenge of spectrum sensing is the receiver uncertainty problem [1] such as practical multipath fading and shadowing, which compromise the detecting performance significantly. Recent research progress shows cooperative spectrum sensing [5]

- W. Zhang is with Western Digital Corporation, Irvine, CA 92614. E-mail:leven2007@gmail.com.

- Y. Guo and Y. Chen are with Department of Electrical \& Computer Engineering, Stevens Institute of Technology, Hoboken, NJ 07030.

E-mail: \{yguo1,yingying.chen\}@stevens.edu.

- H. Liu is with the Department of Computer Information and Technology, Indiana University-Purdue University Indianapolis, Indianapolis, IN 46202. E-mail:leo.hongbo@gmail.com.

- Z. Wang is with the Department of Electrical \& Computer Engineering, University of Toronto, Toronto, ON M5S 3G4, Canada.

E-mail: zhengwang319@gmail.com.

- J. Mitola is with Allied Communications, Boston, MA 02111. E-mail:Joe.Mitola@stevens.edu.

Manuscript received 28 Oct. 2012; revised 5 Dec. 2013; accepted 7 Jan. 2014. Date of publication 23 Feb. 2014; date of current version 5 Dec. 2014.

Recommended for acceptance by X.-Y. Li.

For information on obtaining reprints of this article, please send e-mail to: reprints@ieee.org, and reference the Digital Object Identifier below.

Digital Object Identifier no. 10.1109/TPDS.2014.2307951 is a promising methodology to improve the spectrum sensing performance under shadowing, fading and time-varying wireless channels.

Cooperative spectrum sensing scheme enhances the sensing performance by exploiting the observation diversity of networked and spatially located SUs [5]. Among all the cooperative spectrum sensing methods, centralized cooperative sensing [6] lets all SUs report their measurement information to a centralized fusion center, then a global decision is made at the fusion center. Centralized cooperation requires the entire received data be gathered at one place which may be difficult due to communication constraints [7]. Relay-assisted cooperative sensing [5], [8] is a multi-hop cooperation scheme which makes use of the strong sensing channels and strong reporting channels among the SU network in order to improve the overall performance. The multi-hop communication of the relay-assisted sensing may result in extra power cost and the degradation of sensing data quality through the multi-hop communication paths, since they rely on the sensing channel and report channel which are not perfect [5]. Very recently, bio-inspired consensus scheme is introduced to spectrum sensing in [9], [10] for distributed measurement fusion and soft combining. The consensus-based cooperation features self-organizable and scalable network structure and only needs one-hop communication among local neighbors. However, the existing consensus-based spectrum sensing only ensures equal gain combining (EGC) fusion, which takes the average of each SU's measurements for global decision and neglects the locational measurement quality variations of SU network. Therefore, the existing average consensus-based spectrum sensing scheme is incomparable with centralized methods which can achieve weighted gain combining 
(WGC) fusion and use the weighted average of each SU's measurements according to the measurement quality.

In this paper, we propose a distributed cooperative spectrum sensing scheme based on weighted average consensus algorithm. We adopt weighted average consensus algorithm for measurement fusion of the distributed cooperative spectrum sensing. Weighted average consensus-based fusion allows each SU to choose a weight according to the measurement condition, and the global fused statistic is a soft weighted combining reflecting the measurement quality without centralized fusion center. Compared to the existing average consensus based approaches [9], [10], the proposed method offers better detection performance, and achieves comparable performance with the centralized weighted combining method [11], [12]. With rigorous theoretic proof, the proposed method is robust with respect to dynamic communication channel conditions. The weighted average consensus based spectrum sensing provides a generic distributed weighted combining and applies to both additive white Gaussian noise (AWGN) channels and Rayleigh Fading channels.

The main contribution of this paper has two folds. First, we provide formal convergence analysis of the weighted average consensus under fixed and dynamic communication channels, which advances the theoretical development of consensus algorithms and encompasses average consensus as a special case. In particular, we rigorously prove that temporary communication link failures do not affect the convergence of the weighted average consensus under the jointly connected condition. Second, we apply weighted consensus-based distributed weighted soft combining method in cooperative spectrum sensing. Based on preliminary results presented in our early conference paper [13], we provide a formal treatment of the distributed sensing algorithm in this paper. We obtain closed-form optimal weight design in the distributed weighted combining scheme for the generic additive Gaussian channel approximation, and estimate the convergence rate of the consensus iteration under the assumption that each communication link has an independent probability to fail. We characterize the upper bound of the iteration number of the $\epsilon$-convergence, which indicates all SUs are $\epsilon$ close to the final convergence value in the probability sense. Simulation results show significant improvement of the sensing performance compared to existing consensus-based approaches, and the performance of the distributed weighted design is comparable to centralized weight combining schemes.

We organize the rest of this paper as follows: Section 2 illustrates the energy detector model. Section 3 presents the main results on weighted average consensus-based spectrum sensing, including the algorithm design and distributed weight design. Section 4 presents theorems on the convergence of the proposed weighted average consensus algorithm under fixed and dynamic communication channels, which provide the theoretic foundation of the proposed method. Section 5 discusses simulation results and make comparison with existing approaches. We conclude this study in Section 6.

In the supplementary file, which can be found on the Computer Society Digital Library at http:// doi.ieeecomputersociety.org/10.1109/TPDS.2014.2307951,
Section 1 offers related literature review on cooperative spectrum sensing and consensus algorithms. Section 2 presents related notations and models of the consensusbased graph theory. Section 3 offers further analysis of the proposed spectrum sensing scheme including detection threshold settings and fusion convergence in terms of detection performance. Section 4 presents the proofs for the convergence of the proposed consensus algorithm, and discusses the convergence of the proposed algorithm under random link failure network models. Section 5 shows additional simulation results.

\section{Two Stage Sensing and Energy Detection}

Consensus-based cooperative spectrum sensing adopts a two stage detection scheme: sensing and fusion. For each detection time slot, in the sensing stage, each SU makes measurement of the PU signal using energy detectors. In the fusion stage, each SU communicates with its neighbors using the consensus algorithm to fuse the measurement until convergence. After the fusion convergence, each SU holds the global combined statistic and makes the decision with a global point of view. In this section, we introduce the fundamentals of energy detector and existing centralized weighted combining model of SU networks using energy detection.

\subsection{Sensing and Measurement Stage}

In the sensing stage, we adopt the energy detector [14] because it requires lower design complexity and no priori knowledge of primary users. For the $i$ th $\mathrm{SU}$, the received signal $y_{i}(t)$ is modeled as

$$
y_{i}(t)= \begin{cases}n_{i}(t), & \mathcal{H}_{0} \\ h_{i} s_{i}(t)+n_{i}(t), & \mathcal{H}_{1},\end{cases}
$$

where $\mathcal{H}_{0}$ represents the hypothesis without PU's signal, and $\mathcal{H}_{1}$ represents the hypothesis with PU's signal. In $\mathcal{H}_{1}, h_{i}$ represents gain of the channel, $s_{i}(t)$ is the signal from PU, $n_{i}(t)$ is the additive white Gaussian noise, i.e., $n_{i}(t) \sim$ $\mathcal{N}\left(0, \sigma_{i}^{2}\right)$. We call $n_{i}(t)$ the sensing noises and collect their variances into a vector $\sigma=\left[\sigma_{1}^{2}, \sigma_{2}^{2}, \ldots, \sigma_{n}^{2}\right]^{T}$. Without loss of generality, $s(t)$ and $\left\{n_{i}(t)\right\}$ are assumed to be independent of each other.

According to [15], each secondary user calculates a summary statistic $Y_{i}$ over a detection interval of $m$ samples, i.e., $Y_{i}=\sum_{t=0}^{m-1}\left|y_{i}(t)\right|^{2} \quad i \in \mathcal{I}$ where $m$ is determined from the time-bandwidth product $T W$.

Under AWGN measurement channels, the test statistic of the $i$ th $\mathrm{SU}$ using energy detection is given by $Y_{i}$. Since $Y_{i}$ is the sum of the squares of $m$ Gaussian random variables, it can be shown that $Y_{i} / \sigma_{i}^{2}$ follows a central chi-square $\chi^{2}$ distribution with $m$ degrees of freedom if $\mathcal{H}_{1}$ is true; otherwise, it would follow a non-central $\chi^{2}$ distribution with $m$ degrees of freedom and parameter $\eta_{i}$. That is,

$$
\frac{Y_{i}}{\sigma_{i}^{2}}= \begin{cases}\chi_{m}^{2}, & \mathcal{H}_{0} \\ \chi_{m}^{2}\left(\eta_{i}\right), & \mathcal{H}_{1},\end{cases}
$$

where $\eta_{i}=\frac{E_{s}\left|h_{i}\right|^{2}}{\sigma_{i}^{2}}$ is the local SNR at the $i$ th SU and the quantity 


$$
E_{s}=\sum_{t=0}^{m-1}|s(t)|^{2}
$$

represents the transmitted signal energy over a sequence of $m$ samples during each detection interval. Note that the so-defined local SNR is $m$ times the average SNR at the output of the energy detector, which should be equal to $E_{s}\left|h_{i}\right|^{2} / m \sigma_{i}^{2}$. For convenience, we put all $\eta_{i}$ into the following vector $\eta=\left[\eta_{1}, \eta_{2}, \ldots, \eta_{n}\right]^{T}$. According to the central limit theorem, if the number of samples $m$ is large enough (e.g., $\geq 10$ in practice), the test statistics $Y_{i}$ are asymptotically normally distributed with the mean value

$$
\mathrm{E}\left(Y_{i}\right)=\left\{\begin{array}{cc}
m \sigma_{i}^{2} & \mathcal{H}_{0} \\
\left(m+\eta_{i}\right) \sigma_{i}^{2} & \mathcal{H}_{1}
\end{array}\right.
$$

and the variance

$$
\operatorname{Var}\left(Y_{i}\right)=\left\{\begin{array}{cc}
2 m \sigma_{i}^{4} & \mathcal{H}_{0} \\
2\left(m+2 \eta_{i}\right) \sigma_{i}^{4} & \mathcal{H}_{1} .
\end{array}\right.
$$

Gaussian distribution approximation will facilitate the optimal weights design of the soft weighted combining.

Remark 1. After the first stage of sensing and measurement, each SU enters the second stage of fusion. The fusion is based on the measurement data collected in the sensing stage. We further make the assumption that the channel condition will not change during the data fusion stage. This is not a strong assumption as in the later sections, we will show that our proposed consensus-based fusion algorithm converges very fast.

Due to space limitation, we present preliminaries on graph theory and related notations for consensus-based algorithm in Section 2 of the supplementary file, available online.

\subsection{Centralized Weighted Combining}

Centralized cooperative spectrum sensing combines the measurements of the SUs at a fusion center as [11], [12]

$$
Y_{g}=\sum_{i=1}^{n} \omega_{i} Y_{i}=\omega^{T} Y,
$$

where $\omega=\left[\omega_{1}, \omega_{2}, \ldots, \omega_{n}\right]^{T}, \omega_{i} \geq 0$ is the weighting ratio, $Y=\left[Y_{1}, Y_{2}, \ldots, Y_{n}\right]^{T}$ is the measurement of the CR network.

Assume the reporting channel is noise free and all $\left\{Y_{i}\right\}$ are assumed to be normal random variables, $Y_{g}$ is also normally distributed and has mean

$$
\bar{Y}_{g}=\mathrm{E} Y_{g}=\left\{\begin{array}{cc}
m \sigma^{T} \omega & \mathcal{H}_{0} \\
\left(m \sigma+E_{s} g\right)^{T} \omega & \mathcal{H}_{1},
\end{array}\right.
$$

where

$$
\begin{aligned}
\sigma & =\left[\sigma_{1}^{2}, \sigma_{2}^{2}, \ldots, \sigma_{n}^{2}\right]^{T}, \\
g & =\left[\left|h_{1}\right|^{2},\left|h_{2}\right|^{2}, \ldots,\left|h_{3}\right|^{2}\right]^{T},
\end{aligned}
$$

and the variances under different hypotheses are respectively given by

$$
\begin{aligned}
\operatorname{Var}\left(Y_{g} \mid \mathcal{H}_{l}\right) & =\mathrm{E}\left(Y_{g}-\bar{Y}_{g}\right)^{2} \\
& =\omega^{T} \mathrm{E}\left[\left(Y-\bar{Y}_{\mathcal{H}_{k}}\right)\left(Y-\bar{Y}_{\mathcal{H}_{k}}\right)^{T} \mid \mathcal{H}_{k}\right] \omega
\end{aligned}
$$

where $\bar{Y}=\mathrm{E} Y$ and $l \in\{0,1\}$, specifically, we have

$$
\operatorname{Var}\left(Y_{g} \mid \mathcal{H}_{l}\right)= \begin{cases}\omega^{T} \Sigma_{\mathcal{H}_{0}} \omega & \text { under } \mathcal{H}_{0} \\ \omega^{T} \Sigma_{\mathcal{H}_{1}} \omega & \text { under } \mathcal{H}_{1},\end{cases}
$$

where

$$
\begin{gathered}
\Sigma_{\mathcal{H}_{0}}=2 m \operatorname{diag}^{2}(\sigma), \\
\Sigma_{\mathcal{H}_{1}}=2 m \operatorname{diag}^{2}(\sigma)+4 \operatorname{diag}(\eta) \operatorname{diag}^{2}(\sigma) .
\end{gathered}
$$

With a test threshold $\lambda$, we have $Y_{g} \gtreqless \mathcal{H}_{1} \mathcal{H}_{0} \lambda$ and, the performance of the proposed cooperative spectrum detection scheme can be evaluated using false alarm rate $P_{f}$ and detection rate $P_{d}$, as

$$
P_{f}=Q\left[\frac{\lambda-N \sigma^{T} \omega}{\sqrt{\omega^{T} \Sigma_{\mathcal{H}_{0}} \omega}}\right]
$$

and

$$
P_{d}=Q\left[\frac{Q^{-1}\left(P_{f}\right) \sqrt{\omega^{T} \Sigma_{\mathcal{H}_{0}} \omega}-E_{s} g^{T} \omega}{\sqrt{\omega^{T} \Sigma_{\mathcal{H}_{1}} \omega}}\right] .
$$

Given a fixed false alarm $P_{f}$, maximizing $P_{d}$ in Eq. (14) will yield the optimal weights $\omega$, see [12], where an optimal solution based on modified deflection coefficient are discussed on centralized soft combining.

\section{Spectrum Sensing Using Weighted Average Consensus}

In this section, we present our new consensus-based distributed scheme to achieve the weighted measurement combining through local interactions among SUs, instead of processing the measurements in a centralized fusion center.

\subsection{Measurement Fusion and Detection Using Weighted Average Consensus}

This section introducing weighted average consensus algorithm for the distributed fusion. After the sensing stage, each SU obtains the measurement $Y_{i}$ as the PU's signal energy. For simplicity, we denote the $i^{\text {th }}$ agent's measurement as

$$
x_{i}(0)=Y_{i}, \quad i \in \mathcal{I},
$$

where $x_{i}(0)$ is the initial statistic before the fusion at the iteration $k=0$. The proposed weighted average consensusbased combining scheme is

$$
x_{i}(k+1)=x_{i}(k)+\frac{\alpha}{\delta_{i}} \sum_{j \in N_{i}(k)}\left(x_{j}(k)-x_{i}(k)\right),
$$

where $\delta_{i} \geq 1$ is the weighting ratio according to the channel condition of the $i$ th agent, $\alpha$ is the iteration step size 
satisfying $0<\alpha<\frac{1}{d_{\max }}$, where $d_{\max }$ is the maximum node degree defined in Eq. (2) of the supplementary file, available online, $N_{i}(k)$ denotes neighboring node of the $i$ th SU at time step $k$.

In our approach, each SU keeps sensing and takes measurement based on received signal strength, and simultaneously collects sensing information from its connecting neighbor SUs. It then updates its sensing value iteratively using its own and connecting neighbors $a$ sensing data. As time elapses, the sensing information diffuses through the network, and eventually each SU obtains a consensused value which is the weighted average of the measurements from contributing SUs.

If the SU network communication topologies are jointly connected, all the SUs' decision statistics will reach consensus. The final convergence value is:

$$
x_{i}(k) \rightarrow x^{*}=\frac{\sum_{i=1}^{n} \delta_{i} x_{i}(0)}{\sum_{j=1}^{n} \delta_{i}} \quad \text { as } \quad k \rightarrow \infty, \forall i \in \mathcal{I}
$$

By comparing the decision value $x^{*}$ with a pre-defined threshold $\lambda$, every SU locally obtains the global decision as:

$$
\text { Decision } H=\left\{\begin{array}{lc}
\mathcal{H}_{1}, & x^{*}>\lambda \\
\mathcal{H}_{0}, & \text { otherwise. }
\end{array}\right.
$$

Remark 2. For general detection scenarios, the detection threshold $\lambda$ needs to be pre-calibrated offline using Monte Carlo method. For special case such as AWGN measurement channels, $\lambda$ can be calculated by each SU in a distributed way. Please refer to Section 3.2 of the supplementary file, available online, for detailed analysis.

Remark 3. Compared to the centralized soft combining $Y_{g}=\sum_{i=1}^{n} \omega_{i} Y_{i}$ discussed in (6), the distributed consensus iteration (16) achieves an equivalent combining as

$$
\omega_{i}=\frac{\delta_{i}}{\sum_{i=1}^{N} \delta_{i}}
$$

The final decision statistic $x^{*}$ equals the global combining $Y_{g}$ after the convergence. In the consensus scenario, every SU holds a weighted global decision consensus only through local information exchange without centralized fusion center, which is the major advantage over the centralized combining approach. Meanwhile, the existing average consensus-based combining approach [10], [16], [17] is a special case in our proposed algorithm when $\delta_{i}=1, \forall i \in \mathcal{I}$, the measurement quality difference among SU's are neglected.

Remark 4. From the distributed fusion law (16), each SU only needs to know it's own measurement channel condition and the neighbors to communicate with. The SUs do not need to know the network structure and the number of SUs in the network to run the fusion algorithm, and the final combined value (17) only depends on the weight $\delta_{i}$ set by each SU according to its own measurement quality. In Section 4, we will show the convergence condition is jointly connected SU network structure which is usually satisfied for most SU networks with random link failures.

Remark 5. An important feature of the algorithm (16) is the choice of the weighting factor $\delta_{i}$. If the $i^{\text {th }}$ SU has better measurement channel condition, it sets a larger $\delta_{i}$, which makes the iteration (16) rely less on the local information exchange. On the contrary, an SU with poor measurement channel sets a smaller $\delta_{i}$ and relies more on the information from the network in order to improve the overall performance. Therefore, the statistics value across the whole SU network will be dominated by the SUs with better measurements. In the following Sections 3.2 and 3.3, we provide two different schemes to set the weight $\delta_{i}$ according to different measurement channel conditions, namely, the AWGN channel and Rayleigh fading channel, respectively.

\subsection{Distributed Optimal Weight Design under AWGN Measurement Channel}

In this section, we describe the distributed optimal weight design based on the proposed weighted average consensus algorithm under AWGN measurement channels. First, we consider the combined global statistic in Eq. (6) and obtain the optimized centralized weights $\omega_{o i}$. Using Eq. (19), we obtain the distributed optimal weights $\delta_{o i}$ from $\omega_{o i}$.

Given a false alarm constraint $P_{f}$, the optimal weights can be obtained by maximizing $P_{d}$. Generally speaking, the closed form solution does not exist for maximizing $P_{d}$ in Eq. (14). To give an optimal weights design, we maximizes the deflection coefficient [18] to obtain the centralized near optimal solution as

$$
\omega_{o i}=\frac{\frac{\eta_{i}}{\sigma_{i}^{2}}}{\sum_{i=1}^{n} \frac{\eta_{i}}{\sigma_{i}^{2}}}
$$

where $\omega_{o i}$ denotes the optimal value of $\omega_{i}, \eta_{i}$ is the local SNR defined in Eq. (2.1) and $\sigma_{i}^{2}$ is the variance of the Gaussian noise in the measurement channel. Comparing Eqs. (19) and (20), we obtain the distributed weights as

$$
\delta_{o i}=\frac{\eta_{i}}{\sigma_{i}^{2}}
$$

Remark 6. Because the weighted average consensus ensures the linear combining, the uniform weights should be in a linear form as in Eq. (20). All the $\delta_{o i}$ need to be scaled or saturated to be larger than 1 without affecting the convergence of the consensus iteration under i.i.d. AWGN channel.

To show the optimality of the weight in Eq. (21), we define the deflection coefficient based on the cooperative spectrum sensing settings, as

$$
d^{2}(\omega)=\frac{\left[\mathrm{E}\left(Y_{g} \mid \mathcal{H}_{1}\right)-\mathrm{E}\left(Y_{g} \mid \mathcal{H}_{0}\right)\right]^{2}}{\operatorname{Var}\left(Y_{g} \mid \mathcal{H}_{0}\right)}=\frac{\left(E_{s} g^{T} \omega\right)^{2}}{\omega^{T} \Sigma_{\mathcal{H}_{0}} \omega},
$$

where $\mathrm{E}\left(Y_{g}\right)$ and $\operatorname{Var}\left(Y_{g}\right)$ under the hypothesis $\mathcal{H}_{0}$ and $\mathcal{H}_{1}$ are defined in (7) and (9), respectively. Rewriting Eq. (14) as 


$$
\begin{aligned}
P_{d} & =Q\left[\frac{Q^{-1}\left(P_{f}\right)-\frac{E_{s} T^{T} \omega}{\sqrt{\omega^{T} \Sigma_{\mathcal{H}_{0}} \omega}}}{\sqrt{\frac{\omega^{T} \Sigma_{\mathcal{H}_{1}} \omega}{\omega^{T} \Sigma_{\mathcal{H}_{0}} \omega}}}\right] \\
& =Q\left[\frac{Q^{-1}\left(P_{f}\right)-\frac{E_{s} g^{T} \omega}{\sqrt{\omega^{T} \Sigma_{\mathcal{H}_{0}} \omega}}}{\sqrt{1+\frac{4 \omega^{T} \operatorname{diag}(\eta) \operatorname{diag}^{2}(\sigma) \omega}{\omega^{T} \Sigma_{\mathcal{H}_{0}} \omega}}}\right],
\end{aligned}
$$

where $Q(\cdot)$ denotes the complementary cumulative distribution function. From Eq. (23), we can see that in low SNR channel condition when $\frac{4 \omega^{T} \operatorname{diag}(\eta) \operatorname{diag}^{2}(\sigma) \omega}{\omega^{T} \Sigma_{\mathcal{H}_{0}} \omega} \ll 1$, maximizing $\frac{\left(E_{s} g^{T} \omega\right)^{2}}{\omega^{T} \Sigma_{\mathcal{H}_{0}} \omega}$ will yield a near optimal weights design. We formulate the problem as,

$$
\max _{\omega} d^{2}(\omega), \quad \text { st. } \sum_{i=1}^{N} \omega_{i}=1, \quad \omega_{i}>0, \forall i \in \mathcal{I} .
$$

Solving (24), we can obtain optimal distributed solution using Eq. (19).

Substituting $\omega^{\prime}=\Sigma_{\mathcal{H}_{0}}^{1 / 2} \omega$ into (22) yields

$$
d^{2}(\omega)=\frac{E_{s}^{2} \omega^{T} \Sigma_{\mathcal{H}_{0}}^{-T / 2} g g^{T} \Sigma_{\mathcal{H}_{0}}^{-1 / 2} \omega^{\prime}}{\omega^{\prime T} \omega^{\prime}} .
$$

From Eqn. (22), we know $d^{2}(\omega)$ is non-negative. If we denote the maximum eigenvalue of matrix $\Sigma_{\mathcal{H}_{0}}^{-T / 2} g g^{T} \Sigma_{\mathcal{H}_{0}}^{-1 / 2}$ as $\lambda_{\text {max }}$, we can see from (25) that $d^{2}(\omega)$ reaches its maximum when $\omega^{\prime}$ equals the eigenvector associated with $\lambda_{\text {max }}$. Meanwhile, the matrix $\Sigma_{\mathcal{H}_{0}}^{-T / 2} g g^{T} \Sigma_{\mathcal{H}_{0}}^{-1 / 2}$ is a rank one matrix having the nonzero eigenvalue $\lambda_{\max }=\left\|\Sigma_{\mathcal{H}_{0}}^{-T / 2} g\right\|_{2^{\prime}}^{2}$ and the associated eigenvector $\Sigma_{\mathcal{H}_{0}}^{-T / 2} g$. Let $\omega^{\prime}=\Sigma_{\mathcal{H}_{0}}^{-T / 2} g$, $d^{2}(\omega)$ will achieve the maximum value $E_{s}^{2}\left\|\Sigma_{\mathcal{H}_{0}}^{-T / 2} g\right\|_{2}^{2}$. Therefore, the uniformed optimal weight is

$$
\omega_{o}=\frac{\Sigma_{\mathcal{H}_{0}}^{-1 / 2} \omega^{\prime}}{\mathbf{1}^{T} \Sigma_{\mathcal{H}_{0}}^{-1 / 2} \omega^{\prime}}=\frac{\Sigma_{\mathcal{H}_{0}}^{-1} g}{\mathbf{1}^{T} \Sigma_{\mathcal{H}_{0}}^{-1} g} .
$$

Because $\Sigma_{\mathcal{H}_{0}}$ defined in Eq.(11) is a diagonal matrix, we have

$$
\omega_{o i}=\frac{\frac{\left|h_{i}\right|^{2}}{2 m \sigma_{i}^{4}}}{\sum_{i=1}^{n} \frac{\left|h_{i}\right|^{2}}{2 m \sigma_{i}^{4}}}=\frac{\frac{\eta_{i}}{\sigma_{i}^{2}}}{\sum_{i=1}^{n} \frac{\eta_{i}}{\sigma_{i}^{2}}} .
$$

Using Eq. (19), we can choose $\delta_{o i}=\frac{\eta_{i}}{\sigma_{i}^{2}}$ as a distributed optimal design. Thus, the final consensus value is the near optimal soft weighted combining.

\subsection{Heuristic Weight Design under Rayleigh Fading Channel}

We considered optimal weight design under AWGN channel in the last section. Under different channel conditions, we shall consider different schemes for choosing the weighting parameter $\delta_{i}$. In this section, we discuss the weight design under Rayleigh fading channels, which was first presented in our conference paper [13].

Following [14], [19], under Rayleigh fading, the channel gain $h$ is random and the resulting SNR $\gamma$ follows an exponential distribution. The output $Y$ of each energy detector has the following distributions under hypothesis:

$$
Y \sim\left\{\begin{array}{cc}
\chi_{2 T W}^{2}, & \mathcal{H}_{0} \\
\chi_{2 T W-2}^{2}+Y_{e}, & \mathcal{H}_{1},
\end{array}\right.
$$

where $\chi_{2 T W}^{2}$ and $\chi_{2 T W-2}^{2}$ denote random quantities under central chi-square distributions with mean $2 T W$ and $2 T W-2$, respectively. $Y_{e}$ has an exponential distribution with parameter $2(\bar{\gamma}+1), \bar{\gamma}$ represents the average SNR of the fading channel. The hypothesis $\mathcal{H}_{0}$ and $\mathcal{H}_{1}$ denote the absence and presence of the primary user, respectively. $T W$ is the preset time-bandwidth product of the energy detector which can be set to any integer, we denote $T W=m$ for simplicity.

General closed-form of optimal weight design under Rayleigh fading channel does not exist. We could simply adopt the weights from estimated average channel SNR. From the $\mathcal{H}_{1}$ in (28), each SU could estimate the average SNR from recent measurements as $\bar{\gamma}_{i}=\frac{1}{2 l} \sum_{j=k-l}^{k}\left(Y_{i, j}-2 m\right)$, where $Y_{i, j}$ is the $j$ th measurement of the $i$ th secondary user, $l$ is the estimation window. Setting the weight $\delta_{i}$ in Eq. (16) as

$$
\delta_{i}=\bar{\gamma}_{i}, \quad \forall i \in \mathcal{I},
$$

where $\bar{\gamma}_{i}$ is the estimated average SNR, The final consensus value will be $x_{i}(k) \rightarrow x^{*}=\frac{\sum_{i=1}^{n} \bar{\gamma}_{i} x_{i}(0)}{\sum_{i=1}^{n} \bar{\gamma}_{i}}$ [13].

Remark 7. From Eq. (19), the centralized heuristic weight design is given as $\omega_{i}=\frac{\bar{\gamma}_{i}}{\sum_{i=1}^{n} \bar{\gamma}_{i}}$, which is reported in [20].

\section{Convergence Analysis of the Weighted Average Consensus Algorithms}

In this section, we rigorously prove the convergence of the consensus-based combining algorithm in Eq. (16) under fixed and dynamic communication channel conditions. We further characterize the convergence rate assuming each communication link has a failure probability.

\subsection{Fixed Communication Channel}

Analyzing the convergence of the algorithm (16) under fixed communication channels will bring basic understanding of the weighted consensus algorithm and help the analysis of the algorithm under dynamic communication channels. For convenience, we re-write the algorithm (16) in the following compact form:

$$
x(k+1)=W x(k),
$$

where $x=\left[x_{1}, \ldots, x_{n}\right]^{T}$, and $W$ is defined as

$$
W=I-\alpha \Delta^{-1} L,
$$

where $\Delta=\operatorname{diag}\left\{\delta_{1}, \ldots, \delta_{n}\right\}, L \in \mathbb{R}^{n \times n}$ is the Laplacian matrix defined in Eq. (10). The stepsize $\alpha$ satisfies 


$$
0<\alpha<\frac{1}{d_{\max }}, \quad i \in \mathcal{I} .
$$

The convergence of Eq. (30) depends on the convergence of the infinite matrix product

$$
\lim _{k \rightarrow \infty} W^{k}=\frac{\mathbf{1} \delta^{T}}{\delta^{T} \mathbf{1}},
$$

where

$$
\delta=\left[\delta_{1}, \delta_{2}, \ldots, \delta_{n}\right]^{T},
$$

and $\delta^{T}$ is the left eigenvector of $W$ associated with the eigenvalue 1 . We have the following theorem.

Theorem 1. For the iteration process (16), if the stepsize $\alpha$ satisfies maximum node degree constraint (32), and the elements of matrix $\Delta=\operatorname{diag}\left\{\delta_{1}, \ldots, \delta_{n}\right\}$ satisfy $\delta_{i} \geq 1, \forall i \in \mathcal{I}$, and the communication graph is fixed, then the iteration exponentially converges to $\lim _{k \rightarrow \infty} W^{k} x(0)=\frac{\sum_{i=1}^{n} \delta_{i} x_{i}(0)}{\sum_{i=1}^{n} \delta_{i}} \mathbf{1}$. That is,

$$
x^{*}=\lim _{k \rightarrow \infty} x(k)=\frac{\sum_{i=1}^{n} \delta_{i} x_{i}(0)}{\sum_{i=1}^{n} \delta_{i}} .
$$

Proof. According to Lemma 1 in Section 2.1 of the supplementary file, available online, the Perron matrix $W$ defined in (31) is a primitive matrix [21]. The convergence of (33) and (35) concludes from the famous Perron Frobenius Theorem [21].

Remark 8. Setting weights $\delta_{i}$ in the consensus algorithm makes the information flow rate imbalance between any pair of SU nodes. For any pair of neighboring SUs $\left(v_{i}, v_{j}\right)$, the $i$ th SU has the stepsize $\frac{\epsilon}{\delta_{i}}$, while the $j$ th SU has the stepsize $\frac{\epsilon}{\delta_{j}}$. This makes the network matrix, Laplacian matrix and Perron matrix, asymmetric, and the final convergence value deviates from the average consensus. Setting $\delta_{i} \geq 1, \forall i \in \mathcal{I}$ is a sufficient condition to ensure convergence of the consensus algorithm.

Remark 9. Setting $\Delta=I$, all the weight $\delta_{i}=1, \forall i$, we have $W$ as a symmetric matrix with real eigen spectrum and eigen space. 1 is the simple and largest eigenvalue of $W$, the vector $\mathbf{1}$ and $\mathbf{1}^{T}$ are the associated left and right eigenvectors respectively. The convergence of the consensus iteration is given as

$$
\lim _{k \rightarrow \infty} W^{k} x(0)=\frac{\mathbf{1 1}^{T}}{\mathbf{1}^{T} \mathbf{1}} x(0)=\frac{\sum_{i=1}^{n} x_{i}(0)}{n} \mathbf{1},
$$

which is the average consensus algorithm extensively studied in the literature [22], [23], [24], to name a few.

\subsection{Dynamic Communication Channel}

Realistic SU networks suffer from noise and error interruption or power use constraints. Link failures and dynamic switching communication channels should be considered. In this section, we characterize the conditions for the weighted average consensus convergence on the dynamic communication channels.

For a network of $n$ secondary users, there are a finite number, say a total of $r$, of possible communication graphs.
We denote the set of all possible graphs by $\left\{G_{1}, \ldots, G_{r}\right\}$, and the set of corresponding Laplacian matrices and Perron matrices given by $\left\{L_{1}, \ldots, L_{r}\right\}$ and $\left\{W_{1}, \ldots, W_{r}\right\}$, respectively. We have

$$
W_{s}=I-\alpha \Delta^{-1} L_{s}
$$

for any $1 \leq s \leq r$, where $\Delta=\operatorname{diag}\left\{\delta_{1}, \ldots, \delta_{n}\right\}$. The weighted average consensus algorithm is given by

$$
x(k+1)=W_{s(k)} x(k),
$$

where the indices $s(k)$ are integers and satisfy $1 \leq s(k) \leq r$ for all $k>0$. Here, we use the notion $W_{s(k)}$ to denote the graph sequence in the iteration because the graph sequences could be stochastic or deterministic. We will use $W(k)$ to denote the stochastic case later.

Theorem 2. For the iteration process (38), if the step size $\alpha$ satisfies $0<\alpha<\frac{1}{n}$, where $n$ is the number of the SU nodes in the network, and the elements of matrix $\Delta$ satisfy $\delta_{i} \geq 1, \forall i \in \mathcal{I}$, and the collection of bidirectional communication graphs that occur infinitely often are jointly connected, then the iteration converges to

$$
\lim _{k \rightarrow \infty} x_{i}(k)=\frac{\sum_{i=1}^{n} \delta_{i} x_{i}(0)}{\sum_{i=1}^{n} \delta_{i}}, \quad \forall i \in \mathcal{I} .
$$

Proof. Please refer to Section 4.1 of the supplementary file, available online.

Remark 10. Theorem 2 encompasses the average consensus as a special case when $\delta=I$ and $W_{s(k)}$ are symmetric matrices. For symmetric $W_{s(k)}$, we have $\left\|W_{s(k)}\right\|_{2}=$ $\rho\left(W_{s(k)}\right) \leq 1$, based on which the convergence analysis is given in [22]. For asymmetric $W_{s(k)}$, we adopt the $\mathcal{L}_{\infty}$ norm $\left\|W_{s(k)}\right\|_{\infty}$ for the convergence analysis. Meanwhile, the fixed communication topology, Theorem 1 is a special case when $W_{s(k)}=W, \forall k \geq 0$.

Remark 11. Theorem 2 requires weak long-term connectivity which contains both deterministic and stochastic time-varying graph sequences, and the convergence rate in general may not exist. If we further assume each link has an independent probability to fail, e.g., the link erasure model [25], we can present the estimation of the convergence rate of the consensus iteration in Section 4.2 of the supplementary file, available online.

\section{Simulations AND Discussions}

In this section, we conduct simulations to study the performance of our proposed distributed weighted combining scheme. The simulation setup, evaluation metrics and comparison methodologies are described. We show the convergence of the weighted consensus algorithm, and Monte Carlo simulation [18] is also conducted to evaluate the detection performance of the proposed distributed spectrum sensing scheme.

\subsection{SU Network Setup}

In the simulation, we mainly consider a 10-node SU network as shown in Fig. 1, with both fixed and dynamic 


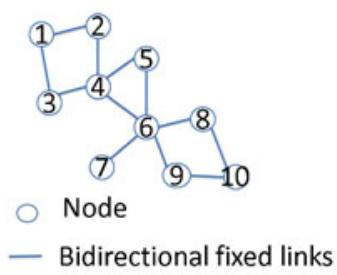

(a)

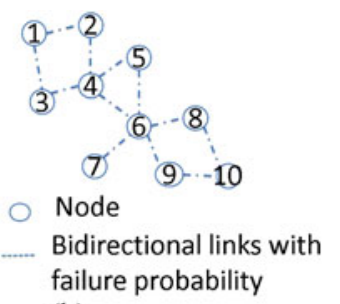

(b)
Fig. 1. Communication network of 10 SUs. [9] (a) fixed communication duplex channels, (b) dynamic communication channels with random link failures.

communication channels. In particular, Fig. 1a shows that 10 SUs communicate with each other through fixed duplex communication channels, and Fig. 1b shows the case of dynamic communication channels which is generated from Fig. 1a by assigning a failure probability 0.4 to each communication link. We use Fig. $1 b$ as a general model for the realistic SU networks suffering from noise and error interruptions. In the simulations shown later, we vary the network size, and consider 20- and 30-node SU networks for the detection performance evaluation. In Section 5 of the supplementary file, available online, the detection performance of 50- and 100-node SU networks are evaluated.

In the sensing stage, all SUs are assumed to be static and have uncorrelated measuring channels with independent fading effects. In the simulation, we mainly consider AWGN and Rayleigh fading channels. According to the energy detector model, each SU generates the measurement $Y_{i}$ of the PU's signal energy under the hypothesis $H_{1}$ in (2) for AWGN channel and $H_{1}$ in (28) for Rayleigh fading channel, respectively. The time-bandwidth product in the energy detector is set to $m=12$. In the Rayleigh fading channel, the estimation window of the average channel SNR estimator is set to $l=2$. In the fusion stage, each SU sets its initial measurement statistic $x_{i}(0)=Y_{i}$ and starts the iterative fusion using algorithm (16) until the final consensus is reached after convergence. The measurement fusion only adopts local communications without a fusion center. In both sensing and fusion stages, all SUs are running the synchronized clock as the same assumption made in other consensusbased schemes [10], [17].

\subsection{Convergence of Consensus Algorithm}

Convergence of the consensus algorithm is crucial in the distributed cooperative spectrum sensing, since the convergence time decides the agility of cognitive radio network sensing capability. Fig. 2 shows the convergence performance of the proposed algorithm with respect to the iteration step under both fixed and dynamic communication channels. The optimal weight is set on each SU according to the its channel conditions. We observe in Fig. 2a that within 30 steps the differences of $x_{i}$ among all the SUs with fixed communication channels are less than $1 \mathrm{~dB}$, indicating the consensus has been reached on the global decision statistics $11.1 \mathrm{~dB}$. Fig. $2 \mathrm{~b}$ shows the convergence of the algorithm under dynamic communication channels with independent link failures, where the failure probability of each link is 0.4 . We observe

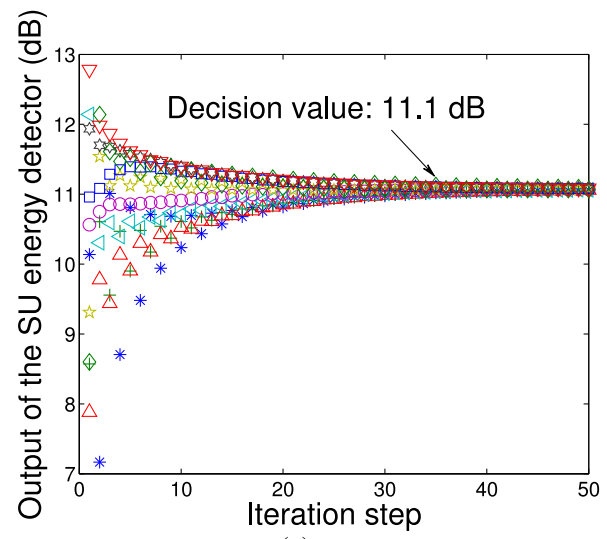

(a)

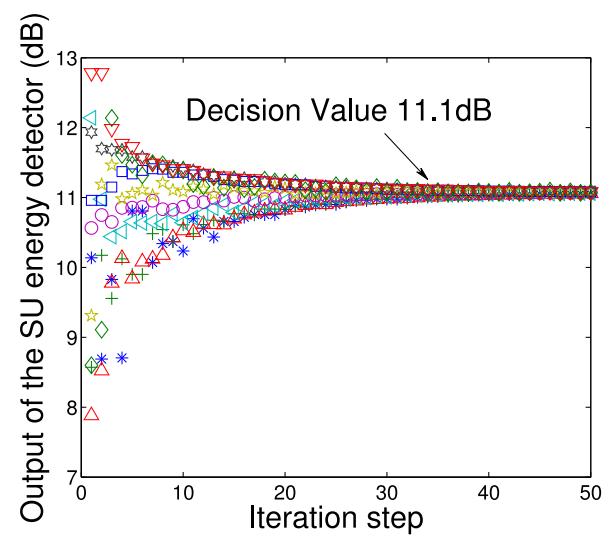

(b)

Fig. 2. Convergence of the proposed consensus algorithm under the 10-node SU network. (a) Fixed communication channels, (b) Dynamic communication channels with independent link failure probability 0.4 .

that the consensus is achieved within 30 iteration steps, which is less than 1 second. As expected, the final convergence is ensured under the random link failure model, but the trajectories of the converging statistics are not as smooth as the case in fixed graph case, due to the temporary link failures. The convergence rate is close but slightly slower than the fixed graph case, which is in accordance with the theoretical conclusion in Remark 4 that it is the spectral gap decides the convergence rate and the random link failure does not necessarily degrades the convergence performance.

\subsection{Metrics and Methodologies}

For comparison, we mainly consider $P_{d}$ (detection probability) and $P_{f}$ (probability of false alarm) as performance metrics, where $P_{d}$ is defined as the probability of decision made on $\mathcal{H}_{1}$ when $\mathcal{H}_{1}$ is true, $P_{f}$ is defined as the probability of decision made on $\mathcal{H}_{1}$ when $\mathcal{H}_{0}$ is true, $\mathcal{H}_{0 / 1}$ are defined in Eq. (1). A high $P_{d}$ will result in high $P_{f}$, which increases the interference to primary users. On the other hand, a low $P_{f}$ will result in low $P_{d}$ and lead to low spectrum utilization. Offering high $P_{d}$ under low $P_{f}$ constraints is challenging for most spectrum seing approaches.

In the performance comparison, we mainly consider the general AWGN and Rayleigh fading channel conditions. Under the AWGN channel condition, we compare our proposed distributed optimal weight gain combining 
(DWGC) discussed in Section 3.2, with the existing equal gain combining [10], OR hard combining (OR) [26], and centralized optimal weighted gain combining [12]. Under the Rayleigh fading channel condition, we compare our distributed weight design based on distributed estimated average channel SNR weighted gain combining (DAWGC) as discussed in Section 3.3, with existing EGC, OR and centralized estimated Average channel SNR weighted gain combining (AWGC) [20] approaches. The (OR) method simulated in this section is 1 out of $n$ detection scheme in which $\mathcal{H}_{1}$ is decided by the largest measurement through the network [26].

To validate the effectiveness of the proposed method, we conduct extensive simulations by varying the measurement channel condition, PU transmission power, and the network size. The threshold $\lambda$ is computed from the false alarm constraints under the hypothesis $H_{0}$ in Eqs. (2) and (28), respectively. The soft combining scheme (DWGC, DAWGC, EGC centralized WGC and AWGC) share the same threshold, since the measurement output under $H_{0}$ is independent of the channel SNR when the primary user signal is absent. The decision threshold of OR hard combining is decided by the largest measurement among the SU network under the hypothesis $H_{0}$.

\subsection{Performance Evaluation}

In this section, we present the simulation evaluation of our proposed method in comparison with existing cooperative spectrum schemes: centralized WGC, EGC and OR methods. We mainly evaluate the detection performance under AWGN channel and Rayleigh fading channels. In the simulation, our approach features the distributed weights choosing by each SU according to its own measurement quality. The weight design for each SU are derived from Equations (21) and (29) for AWGN and Rayleigh fading channels, respectively.

\subsubsection{Receiver Operating Curves (ROC) under AWGN Channels}

In this scenario, we consider the AWGN channel for the evaluation of the proposed DWGC with existing EGC, OR and centralized WGC. Fig. 3a shows the receiver operating curves under AWGN channel for the 10-node SU network shown in Fig. 1, where the channel SNR of the SU network ranges from 0 to $-10 \mathrm{~dB}$. The proposed DWGC achieves the best performance which is comparable to the centralized WGC. As expected, the temporal communication channel failures do not affect the detection performance of the DWGC approach. The EGC approach has a satisfactory performance but the performance is worse than DWGC. The OR scheme performs the worst. Particularly, with the false alarm $P_{f}=0.1$ the DWGC offer the detection probability $P_{d}=0.97$, which is in consistent with the numerical results shown in supplementary file, available online, Section 5.1 Fig. 2(a). EGC and OR give $P_{d}=0.92$ and $P_{d}=0.68$, respectively. Fig. 3a shows clearly the proposed DWGC scheme achieves comparable performance with the centralized WGC and outperforms EGC and OR schemes under AWGN channels.

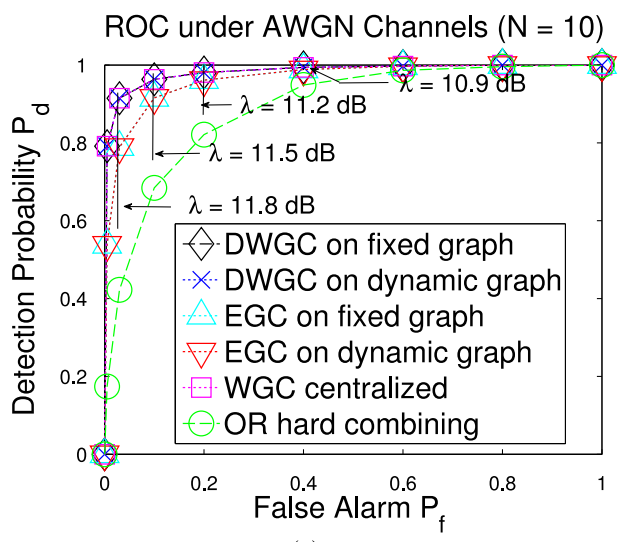

(a)

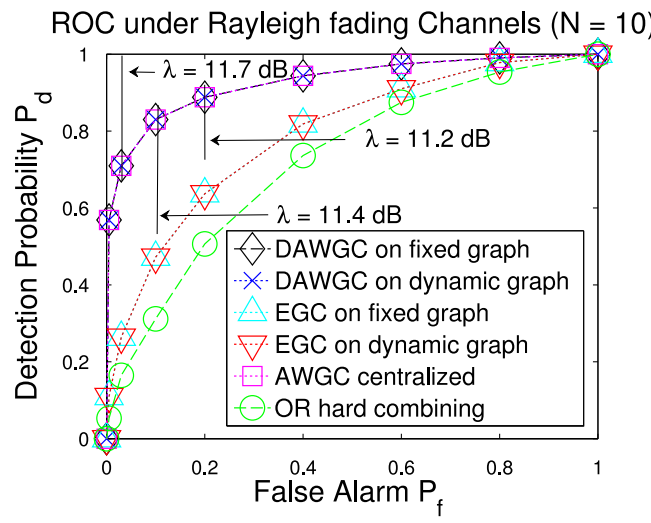

(b)

Fig. 3. (a) ROC of the 10-node SU network under AWGN channels. Channel SNR ranges from 0 to $-10 \mathrm{~dB}$. (b) ROC of the 10-node SU network under Rayleigh fading channels. Average channel SNR $0 \mathrm{~dB}$.

\subsubsection{Receiver Operating Curves under Rayleigh Fading Channels}

In this simulation, we consider the Rayleigh fading channel condition, since Rayleigh fading is inevitable in practice. We compare the detection performance of the proposed DAWGC with EGC, OR and centralized WGC. The average channel SNR is set to $0 \mathrm{~dB}$. From the ROC curves shown in Fig. 3b, we observe that DAWGC has the best performance under both fixed and dynamic communication channels. The DAWGC and centralized AWGC achieve comparable performances. Specifically, when the false alarm is $P_{f}=0.1$, DAWGC achieves detection probability $P_{d}=0.83$, which is the same as the centralized AWGC, while EGC only offers $P_{d}=0.47$ and the OR hard combining is the worst with $P_{d}=0.31$. From Fig. $3 \mathrm{~b}$, we find that the performance degradation of the DWGC and centralized WGC due to Rayleigh fading effects is within 20 percent, while the performance of the EGC and OR-rule degrades more than 50 percent after the Rayleigh fading is considered. The advantage of the proposed method is clear that specific weight setting according to the channel conditions can be implemented to improve the overall performance without a centralized fusion center. It is encouraging that the performance of our proposed approach is comparable with the centralized method and outperforms existing distributed schemes, which do not consider weight on the channel conditions. 


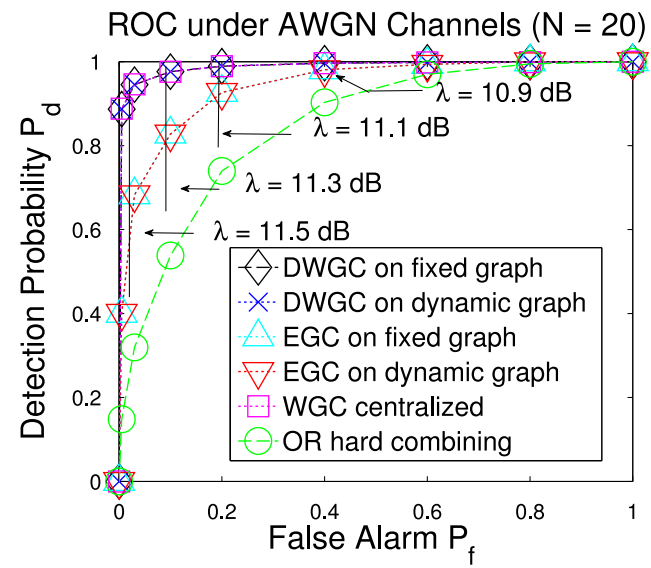

(a)

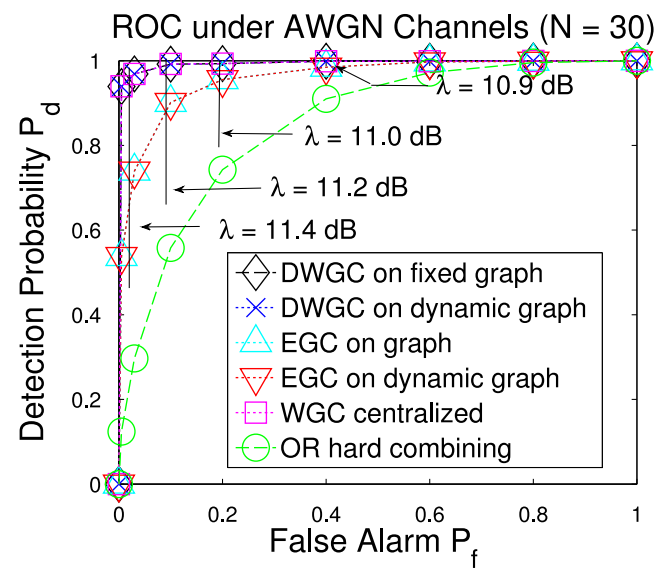

(b)

Fig. 4. ROC under AWGN channels with different network sizes. The channel SNR ranges from 0 to $-15 \mathrm{~dB}$. (a) ROC of a 20 -node SU grid network. (b) ROC of a 30-node SU grid network.

\subsubsection{Receiver Operating Curves with Respect to SU Network Sizes}

We next evaluate the performance of the proposed distributed weighted combining scheme under different network sizes. In Fig. 4, we plot the ROC curves of the proposed DWGC, EGC, OR and centralized WGC under AWGN measuring channel with different SU network sizes. As shown in Figs. 4a and 4b, the SU networks with 20 and 30 nodes are employed, respectively, for performance evaluation under the AWGN channel with identical channel conditions. We observe DWGC achieves the best performance under different network sizes, and have comparable performances with the centralized WGC approach. In particular, when the false alarm $P_{f}$ is set between 0.01 and 0.1, DWGC achieves detection probability above 0.9 . When the network size increases, the detection probability also increases. For both 20- and 30-node cases, detection probability of DWGC is 10 percent higher than the EGC and 25 percent higher than the OR, respectively. Here, the variance of Gaussian noise is fixed at $\sigma_{i}=1, \forall i$, and the measuring channel SNR of the SU network ranges from 0 to $-15 \mathrm{~dB}$. This scenario shows the advantages of the proposed weighted design when false alarm is low, especially more SU nodes in cooperation.

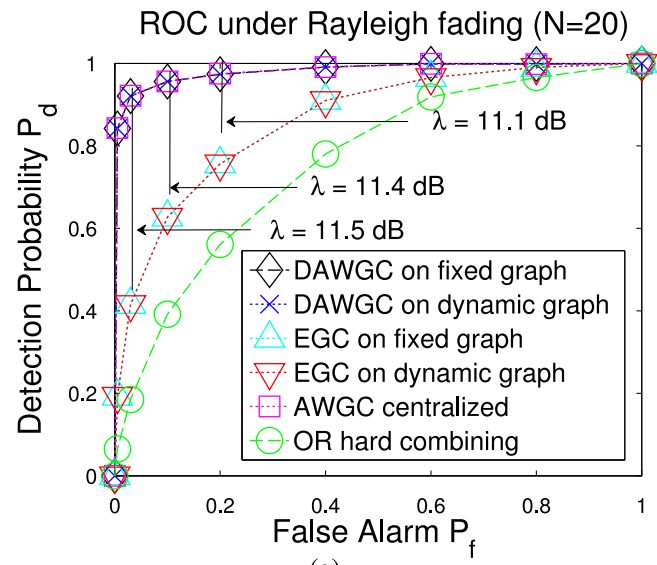

(a)

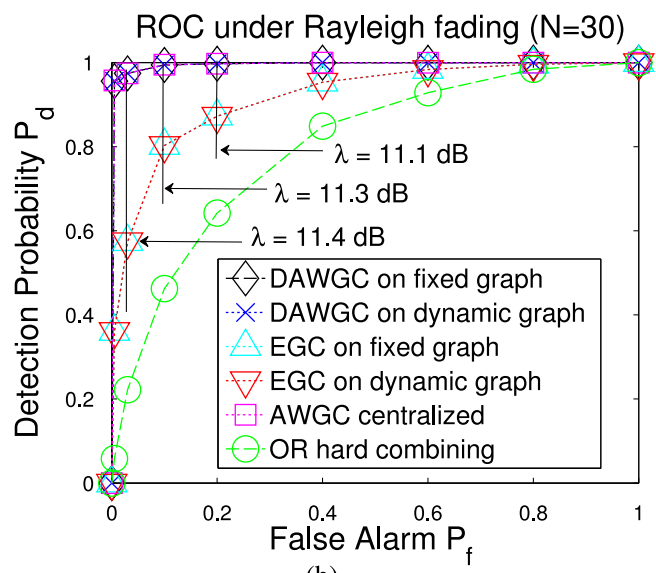

(b)

Fig. 5. ROC under Rayleigh fading channels with different network sizes. Average channel SNR $0 \mathrm{~dB}$. (a) ROC of a 20-node SU grid network. (b) ROC of a 30-node SU grid network.

In Fig. 5, we plot the ROC curves for DAWGC, EGC, OR, centralized AWGC under Rayleigh fading channel with network sizes with 20 and 30 nodes, respectively. We observe that the proposed DAWGC method achieves the best performance in both cases, and the detection probability is comparable to the centralized AWGC. Clearly, the performance of DAWGC is much less affected by the Rayleigh fading than the EGC and OR hard combining. Particularly, when the false alarm is set between 0.005 and 0.1, DAWGC achieves the highest detection probability over 0.8 in the 20-node case and over 0.9 in the 30-node case, respectively. In contrast, when the false alarm is set at 0.005 , the detection probability of EGC is below 0.4 in both 20- and 30-node cases, and OR hard combining performs the worst with detection probability below 0.3 . This scenario demonstrates the advantages of our proposed DAWGC under Rayleigh fading channels with low false alarm rates. DAWGC with distributed weights based on estimated channel SNR is more sensitive to the network size changes, and maintains much higher detection probability under the low false alarm rates, compared to other existing distributed spectrum sensing methods.

In summary, we clearly observe that the proposed distributed consensus-based weighted design outperforms the existing distributed combining approaches significantly, and achieves comparable performance with the centralized 
weighted combining scheme. The proposed distributed optimal weights design can incorporate specific weights according to different channel conditions, and exhibits clear advantages in extensive simulations with respect to channel fading, low PU transmission power, low false alarm rate and the network size variation.

\section{Conclusion}

In this paper, we develop a distributed weighted combining scheme for cooperative spectrum sensing in cognitive radio networks. The proposed method is based on the weighted average consensus algorithm for both fixed and time-varying network graphs. Through the weighted local fusion iteration, each secondary user derives the global decision statistic from the weighted soft measurement combining throughout the network to achieve distributed cooperative spectrum sensing. When the weights are appropriately chosen, the detection performance of the proposed scheme is comparable to the performance of centralized optimal weighted combining scheme and outperforms existing distributed equal gain combining schemes. The authors are pursuing several future work directions. Adopting the distributed consensus estimator algorithm [27], [28] can achieve weighted combined decision statistics in real-time without waiting for the consensus convergence for the decision making. The other direction is to study the effects of different communication link dynamics [29] on the decision performance. More promising work focuses on the distributed environment mapping [30] using weighted consensus algorithms.

\section{ACKNOWLEDGMENTS}

The authors would like to acknowledge the support by the National Science Foundation under Grants CNS-1318748, IIS-1218155, and CNS-0954020.

\section{REFERENCES}

[1] J. Mitola, "An integrated agent architecture for software defined radio," Ph.D. dissertation, The Royal Institute of Technology, KTH, Stockholm, Sweden, 2000.

[2] I. F. Akyildiz, W.-Y. Lee, M. C. Vuran, and S. Mohanty, "Next generation/dynamic spectrum access/cognitive radio wireless networks: A survey," Comput. Netw., vol. 50, pp. 2127-2159, 2006.

[3] J. Ma, G. Li, and B. H. Juang, "Signal processing in cognitive radio," in Proc. IEEE, vol. 97, no. 5, pp. 805-823, May 2009.

[4] T. Yucek and H. Arslan, "A survey of spectrum sensing algorithms for cognitive radio applications," IEEE Commun. Surveys Tuts., vol. 11, no. 1, pp. 116-130, First Quarter, 2009.

[5] I. F. Akyildiz, B. F. Lo, and R. Balakrishnan. (2011). Cooperative spectrum sensing in cognitive radio networks: A survey, Phys. Commun., vol. 4, no. 1, pp. 40-62, [Online]. Available: http:// www.sciencedirect.com/science/article/B8JDV-51RJDCF-1/2/ cd139bcf 557e7245930a17d0dca26402.

[6] K. B. Letaief and W. Zhang, "Cooperative communications for cognitive radio networks," Proc. IEEE, vol. 97, no. 5, pp. 878-893, May 2009.

[7] S. Mishra, A. Sahai, and R. Brodersen, "Cooperative sensing among cognitive radios," in Proc. IEEE Int. Conf. Commun., 2006, vol. 4, pp. $1658-1663$.

[8] W. Zhang and K. Letaief, "Cooperative spectrum sensing with transmit and relay diversity in cognitive radio networks," IEEE Trans. Wireless Commun., vol. 7, no. 12, pp. 4761-4766, Dec. 2008.

[9] F. Yu, M. Huang, and H. Tang, "Biologically inspired consensusbased spectrum sensing in mobile ad hoc networks with cognitive radios," IEEE Netw., vol. 24, no. 3, pp. 26-30, May/Jun. 2010.
[10] Z. Li, F. R. Yu, and M. Huang, "A distributed consensus-based cooperative spectrum-sensing scheme in cognitive radio," IEEE Trans. Veh. Technol., vol. 59, no. 1, pp. 383-393, Jan. 2010.

[11] J. Ma, G. Zhao, and Y. Li, "Soft combination and detection for cooperative spectrum sensing in cognitive radio networks," IEEE Trans. Wireless Commun., vol. 7, no. 11, pp. 4502-4507, Nov. 2008.

[12] Z. Quan, S. Cui, and A. Sayed, "Optimal linear cooperation for spectrum sensing in cognitive radio networks," IEEE J. Select. Topics Signal Process., vol. 2, no. 1, pp. 28-40, Feb. 2008.

[13] W. Zhang, Z. Wang, Y. Guo, H. Liu, Y. Chen, and J. Mitola III, "Distributed cooperative spectrum sensing based on weighted average consensus," in Proc. IEEE Global Telecommun. Conf., Dec. 2011, pp. 1-6.

[14] M. Alouini, F. F. Digham, and M. K. Simon, "On the energy detection of unknown signals over fading channels," IEEE Trans. Commun., vol. 55, no. 1, pp. 21-24, Jan. 2007.

[15] H. Urkowitz, "Energy detection of unknown deterministic signals," in Proc. IEEE, vol. 55, no. 4, pp. 523-531, Apr. 1967.

[16] M. E. Yildiz, T. C. Aysal, and K. E. Barner, "In-network cooperative spectrum sensing," in EURASIP Eur. Signal Process. Conf., Glasgow, Scotland, 2009, pp. 1903-1907.

[17] S. Kar, S. Aldosari, and J. Moura, "Topology for distributed inference on graphs," IEEE Trans. Signal Process., vol. 56, no. 6, pp. 2609-2613, Jun. 2008.

[18] S. M. Kay, Fundamentals of Statistical Signal Processing: Estimation Theory. Upper Saddle River, NJ, USA: Prentice-Hall, 1993.

[19] V. Kostylev, "Energy detection of a signal with random amplitude," in Proc. IEEE Int. Conf. Commun., 2002, vol. 3, pp. 1606-1610.

[20] F. E. Visser, G. J. Janssen, and P. Pawelczak, "Multinode spectrum sensing based on energy detection for dynamic spectrum access," IEEE Veh. Technol. Conf., pp. 1394-1398, 2008.

[21] M. Carl, Matrix Analysis and Applied Linear Algebra. Philadelphia, PA, USA: SIAM, 2000.

[22] L. Xiao, S. Boyd, and S. Lall, "A scheme for robust distributed sensor fusion based on average consensus," in Proc. 4th Int. Symp. Inform. Process. Sens. Netw., 2005, pp. 63-70.

[23] L. Xiao and S. Boyd, "Fast linear iterations for distributed averaging," Syst. Control Lett., vol. 53, pp. 65-78, 2004.

[24] S. Kar, S. Aldosari, and J. Moura, "Topology for distributed inference on graphs," IEEE Trans. Signal Process., vol. 56, no. 6, pp. 2609-2613, Jun. 2008.

[25] S. Kar and J. Moura, "Sensor networks with random links: Topology design for distributed consensus," IEEE Trans. Signal Process., vol. 56, no. 7, pp. 3315-3326, Jul. 2008.

[26] A. Ghasemi and E. Sousa, "Collaborative spectrum sensing for opportunistic access in fading environments," in Proc. 1st IEEE Int. Symp. New Frontiers Dyn. Spectrum Access Netw., 2005, pp. 131-136.

[27] S. Li and Y. Guo, "Distributed consensus filter on directed graphs with switching topologies," in Proc. Amer. Control Conf., 2013, pp. 6151-6156.

[28] S. Li and Y. Guo, "Dynamic consensus estimation of weighted average on directed graphs," Int. J. Syst. Sci., pp. 1-15, 2013.

[29] X. Ma, S. Djouadi, and H. Li, "State estimation over a semi-Markov model based cognitive radio system," IEEE Trans. Wireless Commun., vol. 11, no. 7, pp. 2391-2401, Jul. 2012.

[30] R. Kong, W. Zhang, and Y. Guo, "Distributed estimation and tracking for radio environment mapping," Accepted by American Control Conference 2014, to appear.

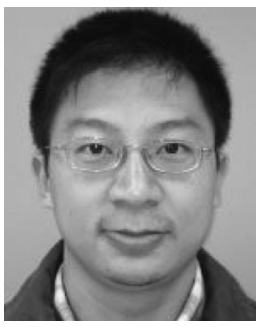

Wenlin Zhang received the BEng degree in automation from the University of Science and Technology of China, Hefei, Anhui, China, and the PhD degree from the Stevens Institute of Technology, Hoboken, NJ. He is currently a staff servo engineer at Western Digital Corporation, Irvine, CA. His research interests include nonlinear control, adaptive control, cooperative control, cognitive radio networks. 


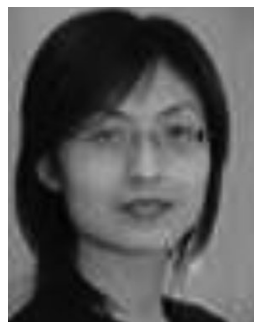

Yi Guo received the BS and MS degrees from the Xi'an University of Technology, China, in 1992 and 1995, respectively, and the PhD degree from the University of Sydney, Australia, in 1999, all in electrical engineering. She is currently an associate professor in the Department of Electrical and Computer Engineering at the Stevens Institute of Technology. She was a postdoctoral research fellow at Oak Ridge National Laboratory from 2000 to 2002, and a visiting assistant professor at the University of Central Florida from 2002 to 2005. Since 2005, she has been with the Stevens Institute of Technology. Her main research interests include autonomous mobile robotics, distributed sensor networks, and nonlinear control systems. She is an associate editor of IEEE Robotics and Automation Magazine. She is a senior member of the IEEE.

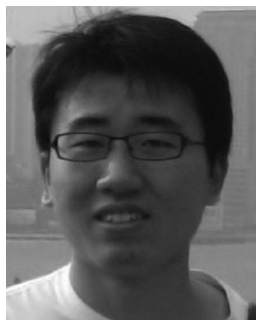

Hongbo Liu received the $\mathrm{PhD}$ degree in electrical engineering from the Stevens Institute of Technology. He is currently an assistant professor in the Department of Electrical and Computer Engineering of IUPUI. His research interests include mobile and pervasive computing, cyber security and privacy, and smart grid. He received the Best Paper Award from ACM MobiCom 2011 and the Best Paper Runner-up Award of IEEE CNS 2013.

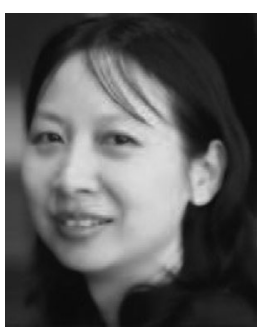

Yingying (Jennifer) Chen received the $\mathrm{PhD}$ degree in computer science from Rutgers University. She is currently an associate professor in the Department of Electrical and Computer Engineering at the Stevens Institute of Technology. Her research interests include cyber security and privacy, mobile and pervasive computing, and mobile healthcare. She has published more than 80 journals and referred conference papers in these areas. Prior to joining Stevens, she was with Alcatel-Lucent. She received the NSF CAREER Award and Google Research Award. She also received NJ Inventors Hall of Fame Innovator Award. She received the Best Paper Award from ACM International Conference on Mobile Computing and Networking (MobiCom) 2011. She also received the IEEE Outstanding Contribution Award from IEEE New Jersey Coast Section each year 2005-2009. Her research has been reported in numerous media outlets including MIT Technology Review, Wall Street Journal, and National Public Radio. She is on the editorial boards of IEEE Transactions on Mobile Computing, IEEE Transactions on Wireless Communications, and IEEE Network Magazine.

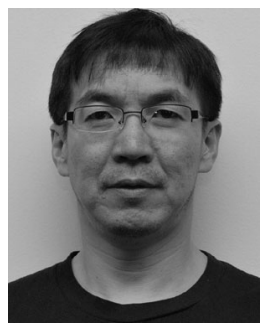

Zheng Wang received the $\mathrm{PhD}$ degree from the Department of Mechanical and Manufacturing Engineering, University of Calgary in 2008. He was a postdoctoral research fellow at the Stevens Institute of Technology from 2009 to 2011 , and then at the University of Toronto from 2011 to 2013. His research interests are in nonlinear control, multi-agent systems, neural network and machine learning.

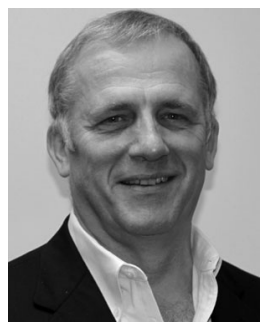

Joseph Mitola III received the BS degree in EE from Northeastern University, Boston, MA, in 1971, MSE degree in stochastic optimal control from The Johns Hopkins University, Baltimore, MD, in 1974, licentiate and doctorate degree in teleinformatics from the KTH Royal Institute of Technology, Stockholm, Sweden, in 2000. Earlier in his career (1974-1993) he held positions of technical leadership with E-Systems, Harris Corporation, Advanced Decision Systems, and ITT Corporation. He began his career as an engineering student assistant (1967) and engineer (69-74) with DoD. Recognized globally as "the Godfather" of software radio and cognitive radio technologies on which dynamic spectrum access (DSA) and spectrum sharing are based, he founded Mitola's STATISfaction for research in flexible commercial radio systems in 1993. STATISfaction's publications include the landmark Software Radio Architecture (Special Issue of the IEEE Communications Magazine, May 1995; Wiley, 2000) and the first Cognitive Radio Architecture (KTH, 1998; Wiley, 2006). Since 2012 through STATISfaction, he has been supporting a wide variety of nonconflicted government and private entities. $\mathrm{He}$ is a widely sought keynote speaker, expert witness, and technical innovator in next generation national security and commercial information systems. He was a vice president for the Research Enterprise of Stevens Institute of Technology, Hoboken, $\mathrm{NJ}$, where he led faculty teams in academic research in critical national technologies (2008-2012). In his 15 years with The MITRE Corporation (1993-2008), he was the chief scientist of MITRE's DoD FFRDC, special assistant to the Director of the US Defense Advanced Research Projects Agency (DARPA), special assistant to the Deputy Director of the US National Security Agency (NSA) for trustable cognitive systems, technical director of Modeling and Simulation, DARPA program manager, and the technical advisor to the Executive Office of the President of the United States.

$\triangleright$ For more information on this or any other computing topic, please visit our Digital Library at www.computer.org/publications/dlib. 